\title{
Aula invertida en el aprendizaje de los métodos numéricos en estudiantes de Ingeniería Civil
}

\author{
Flipped Classroom in the learning of numerical methods in civil \\ engineering students \\ Maximiliano Asís-López $^{1}$ (iD) Vladimir Rodríguez-Sabino ${ }^{1}$ (D) Efracio Asís-López² \\ ${ }^{1}$ Universidad Nacional Santiago Antúnez de Mayolo. Huaraz, Perú \\ ${ }^{2}$ Universidad Nacional Mayor de San Marcos. Lima, Perú
}

Recibido: 06/10/2021 Revisado: 05/11/2021_Aceptado: 12/11/2021 Publicado: 15/01/2022

\section{RESUMEN}

Son numerosos los trabajos que señalan al aula invertida (flipped classroom) como una metodología de clase que aporta beneficios en el proceso de enseñanza-aprendizaje, sobre todo relacionados con la motivación y satisfacción del alumno. Este artículo presenta la planificación y la aplicación del modelo pedagógico "Flipped Classroom" para la enseñanza-aprendizaje en el curso de métodos numéricos. Durante la investigación se realizaron videos tutoriales sobre los contenidos del curso, los cuales fueron elaborados previamente para luego ser presentados a los estudiantes de la Escuela Profesional de Ingeniería Civil de la Universidad Nacional Santiago Antúnez de Mayolo que cursaban la asignatura de Métodos Numéricos. Se analizó la valoración del alumnado y el posible impacto de esta metodología en la calificación de los estudiantes. Los resultados obtenidos, expresan que los alumnos consideran la Clase Invertida como una metodología más divertida y motivadora que la clase tradicional; además, se observa una diferencia en las calificaciones obtenidas por los estudiantes que aprendieron con la metodología de aula invertida y con aquellos que lo hicieron mediante una metodología tradicional. Palabras clave: Aprendizaje activo; aprendizaje electrónico; estrategias de aprendizaje; usos de la tecnología en la educación; métodos de enseñanza.

\section{ABSTRACT}

There are numerous studies that point to the flipped classroom as a classroom methodology that provides benefits in the teaching-learning process, especially related to student motivation and satisfaction. This article presents the planning and application of the "Flipped Classroom" pedagogical model for teaching-learning in the course of numerical methods. During the investigation, tutorial videos were made on the course contents, which were previously prepared and then presented to the students of the Professional School of Civil Engineering of the Santiago Antúnez de Mayolo National University who were taking the subject of Numerical Methods. 
The students 'assessment and the possible impact of this methodology on the students' qualification was analyzed. The results obtained express that the students consider the Inverted Class as a more fun and motivating methodology than the traditional class; Furthermore, a difference is observed in the grades obtained by the students who learned with the flipped classroom methodology and with those who did so through a traditional methodology.

Keywords: Active learning; electronic learning; learning strategies; technology uses in education; teaching methods.

\section{INTRODUCCIÓN}

El avance de las tecnologías de Información y la Comunicación (TIC) ha incorporado cambios en la forma de generar, adquirir y compartir los conocimientos; en el aspecto educativo ha permitido incorporar nuevas metodologías que refuerzan el proceso de enseñanza y aprendizaje (García, 2020), por consiguiente, las TIC promueven el aumento de pensamiento crítico, autónomo y creativo en los estudiantes, facilitando su desenvolvimiento social, económico y cultural (Bergmann y Sams, 2014). Además, en la actualidad el uso de las tecnologías por parte de los estudiantes va en aumento y por consiguiente su forma de vida transcurre entre el entorno virtual y el real (Tourón y Santiago, 2015). Trasladar ese pensamiento al aula, no es más que seguir el proceso evolutivo, y plantear a su vez conexiones entre el aprendizaje formal y no formal. Además se ha demostrado que las videoconferencias existentes, como Khan Academy o las charlas TED, son útiles para minimizar el aburrimiento y distracción (Gilboy et al., 2015).

Actualmente las metodologías de aprendizaje activos emplean las TIC, las cuales ayudan a mejorar la práctica docente; entre las que se encuentran la propuesta de Bergmann y Sams (2014), denominado aula invertida(Flipped Classroom), que consiste en que el alumno previamente estudie los conceptos a tratar en la clase por medio de diversas herramientas que el docente facilita, como vídeos o audios digitales; entre tanto el tiempo de clase estará destinado a fomentar otros procesos, como la discusión, aclaratoria de dudas, puesta en práctica de los conocimientos adquiridos para la resolución de problemas, debates y entre otras actividades; que estimulan el intercambio de ideas y retroalimentación del profesor en el aula (García, Porto y Hernández, 2019; Rivera y García, 2018) y permite al profesor desenvolverse en un ambiente de atención a determinados conocimientos, a la interpretación de los conocimientos para atender dudas y/o detalles de comprensión de los estudiantes para llevar a todos los participantes en el aula a su aplicación (Perdomo, 2016).

El enfoque del aula invertida, se ha vuelto popular en los últimos tiempos en muchas instituciones educativas debido a los avances en las tecnologías educativas, la presión para mejorar el rendimiento de los estudiantes, la voluntad de desafiar los métodos de enseñanza establecidos y presiones dentro de las universidades (Carpenter, Blythe, Sweet, Winter y Bunnell, 2015; Dodds, 2015). El enfoque de aprendizaje del aula invertida tiene el potencial de brindar a los educadores oportunidades de maximizar y aumentar la calidad de la instrucción presencial, ya que se les pide a los estudiantes que asistan a la clase habiendo ya participado con los materiales del curso. Sin embargo, es importante considerar las opiniones de los estudiantes sobre el enfoque para garantizar los mejores 
resultados para los estudiantes y su aprendizaje. Asimismo, Tourón y Santiago (2015) mencionan que la metodología de aula invertida es una herramienta eficaz para el desarrollo de competencias de los alumnos más destacados y permite enseñar de acuerdo al tiempo en que puedan tardar en adquirir el conocimiento, lo que supone una personalización superior para cada uno de los participantes.

En la metodología de aula invertida, es necesario que el docente tome rol de orientador, esto es, cambiar el rol de expositor de conocimientos por el de asesor para así transferir el protagonismo a los estudiantes (Perdomo, 2016; Little, 2015); asimismo el estudiante debe tomar un rol más activo durante su aprendizaje y no ser un simple receptor de contenidos, es decir, ser un productor del conocimiento (Bergmann y Sams, 2014). De esta forma, la metodología de aula invertida hace un gran cambio de roles entre profesores y los alumnos según las metodologías tradicionales, ya que presenta diversas ventajas que potencia el aprendizaje activo, el trabajo colaborativo y el trabajo mediante proyectos entre otros (Novak et al., 2017; Arráez et al., 2018; Rotellar y Cain, 2016).

Los resultados cualitativos y cuantitativos indicaron que el formato de aula invertida generó mayores efectos de aprendizaje y disfrute en el aula, fomentó el aprendizaje autorregulado de los estudiantes, mejoró la interacción grupal, estimuló la actividad grupal y garantizó un comportamiento de aprendizaje más sinérgico (Mastmeyer 2020; Fox-Turnbull, Docherty y Zaka, 2018).

Por otro lado, la metodología aula invertida es un modelo de enseñanza interactivo que se ha introducido con éxito en la enseñanza de matemáticas en las universidades; en materias tales como en matemáticas aplicadas, matemáticas discretas, algebra, topología, algebra lineal y análisis (Maciejewski, 2015; Petrillo, 2016). La aplicación de la metodología de aula invertida está respaldada por la evidencia de que los estudiantes se desempeñan mejor en las clases de aprendizaje activo en comparación con las clases magistrales tradicionales en las materias de ciencia, tecnología, ingeniería y matemáticas (Freeman et al., 2014). Además, los videos parecen ser tan buenos como las conferencias ordinarias cuando se trata de presentar el contenido del curso (DeLozier y Rhodes, 2016).

Teniendo en cuenta la emergencia sanitaria a nivel global, somos conscientes de la transición repentina a la modalidad virtual que ha tenido que adoptarse con el fin de dar continuidad al servicio educativo. Las mejoras en la accesibilidad y la velocidad de Internet han ampliado el alcance global de la educación a una escala sin precedentes en todos los niveles, desde preescolar hasta estudios de posgrado. Los alumnos con dispositivos portátiles y acceso a Internet las 24 horas del día, los 7 días de la semana ya no están limitados al horario escolar. De hecho, estos estudiantes pueden acceder a la información que necesitan donde y cuando quieran. Hoy sabemos que la actitud y las prácticas de los profesores que limitan el uso de la tecnología en el aula pueden obstaculizar el aprendizaje centrado en el alumno (Bolat, 2016). Los esfuerzos para contrarrestar estos obstáculos han llevado a su vez a la popularización de conceptos radicales como el aula invertida (Filiz y Kurt, 2015; Torun y Dargut, 2015). El aula invertida se centra en la idea de que, para lograr el éxito en la educación, el entorno de aprendizaje debe reorganizarse de acuerdo con las necesi- 
dades de los estudiantes. De hecho, la mayoría de los sistemas educativos ahora enfatizan el aprendizaje centrado en el estudiante, valoran las diferencias individuales y exigen que el plan de estudios se prepare en consecuencia. El objetivo principal es comprender las necesidades de las personas e incluirlas activamente en todas las actividades educativas.

Desde este punto de vista, viendo los resultados beneficiosos a nivel educativo y el aumento de trabajos de investigación basados en la metodología de aula invertida, se elige esta metodología para realizar un proyecto de investigación en el que se planteó llevar a cabo una propuesta de implementación de la metodología aula invertida en la asignatura de Métodos Numéricos de la Facultad de Ingeniería Civil de la Universidad Santiago Antúnez de Mayolo. El objetivo de este trabajo es analizar el grado de satisfacción y las percepciones de los estudiantes de la Escuela Profesional de Ingeniería Civil de la Universidad Nacional Santiago Antúnez de Mayolo en el uso de la metodología aula invertida en el curso de Métodos Numéricos.

\section{MATERIALES Y MÉTODOS}

\section{Participantes y contexto}

La población objeto de estudio son los estudiantes de la Facultad de Ingeniería Civil de la Universidad Nacional Santiago Antúnez de Mayolo. En este caso se optó por un muestreo incidental o casual, no aleatorio, seleccionando 32 estudiantes que cursaban dicha asignatura de Métodos Numéricos en un semestre del año lectivo 2020.
La metodología de flipped classroom se introdujo en el momento donde la Universidad Nacional Santiago Antúnez de Mayolo comenzó a ofrecer la enseñanza en forma virtual a causa de la pandemia de covid 19 .

El curso de Métodos Numéricos fue impartido durante dos años consecutivos, su duración fue de 16 semanas por 5 horas a la semana, realizado con el mismo docente; el primer y segundo año se impartió a través de una enseñanza tradicional y el tercer año con la metodología de clase invertida. Los temas se presentaron en el mismo orden y las evaluaciones fueron similares en ambos años, cada una con aproximadamente el mismo número, tipo y dificultad de preguntas.

\section{Fase de diseño de recurso de aprendizaje}

Tomado de base la programación académica de la asignatura, se crearon los videos en formato mp4, como se muestra en la figura 1, en las que se explicó los contenidos de la asignatura de métodos numéricos, apoyándose de paquetes matemáticos y software como: Octave, Geogebra, Matlab, Camtasia, Microsoft Teams y Latex; de modo que resultasen interesantes y atractivos para los estudiantes; asimismo, utilizando como plataforma de recepción un canal de YouTube elaborado para tal fin y para que los estudiantes desarrollen los aprendizajes esperados fuera del aula (clases asincrónicas). Este material fue ampliado cada siete días, de acuerdo con el avance del temario del curso, a la vez que los tutoriales ya trabajados estuvieron siempre disponibles para volver a ser revisados en cualquier momento por los alumnos. Además se tuvo clases sincrónica a través de la plataforma Microsoft Teams. 

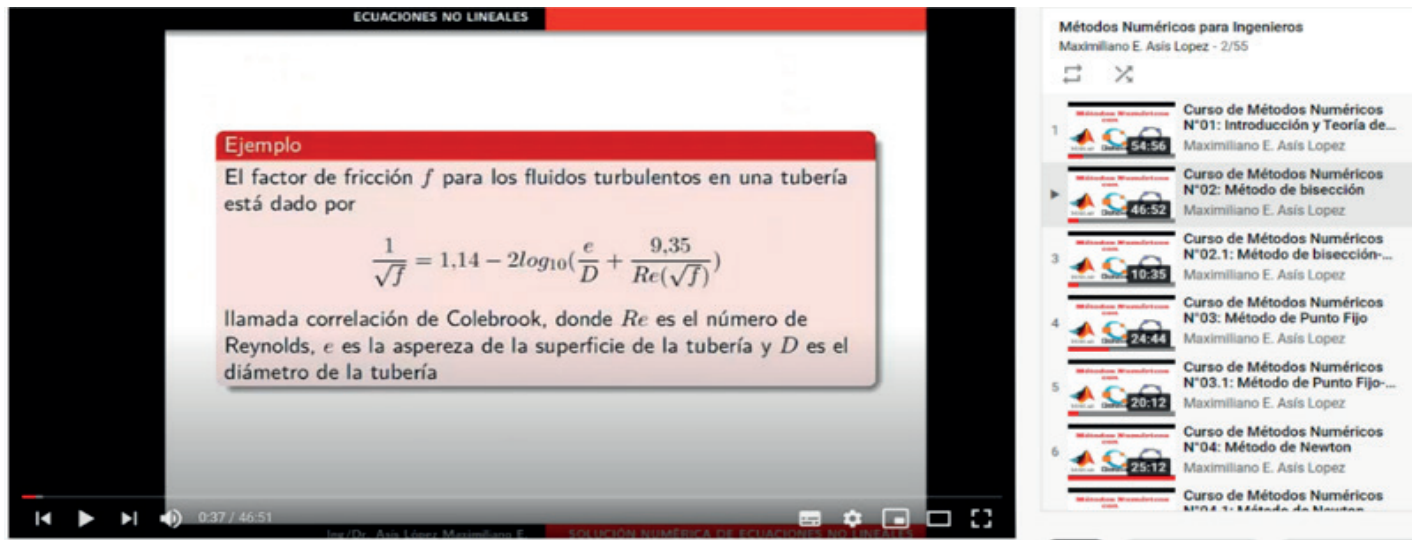

Figura 1. Diseño de videos para la aplicación de aula invertida.

\section{Técnicas e instrumentos}

Se utilizaron dos instrumentos para obtener información sobre la experiencia del aula invertida en el proceso de enseñanza y aprendizaje.

\section{La Encuesta}

Para recabar las percepciones de los estudiantes sobre la experiencia vivida en la aplicación de la metodología de enseñanza Flipped classroom en el curso de métodos numéricos, se utilizó un cuestionario en línea que consistía en un conjunto de 7 ítems que los alumnos debían responder atendiendo a una escala de Lickert, con una valoración de 1 a 5 que corresponde a las etiquetas verbales de 1= Muy en desacuerdo; $2=$ en desacuerdo; $3=$ neutral; $4=$ De acuerdo y $5=$ Totalmente de acuerdo. $\mathrm{La}$ encuesta se aplicó en la última sesión de clase.

\section{Rúbrica}

Los datos de desempeño se basaron en las calificaciones obtenidas de las diferentes actividades realizadas durante el desarrollo del curso en el año 2020, precisamente de las prácticas calificadas, las investigaciones formativas y el examen final del curso. Además, se usó los datos de desempeño obtenidos en año anterior evaluado con las misma rubrica y las mismas actividades realizadas, pero el desarrollo del curso fue con la metodológica presencial en el curso. Para la evaluación de las actividades desarrolladas en el curso, se utilizó la plataforma virtual Moodle de la Universidad Nacional Santiago Antúnez de Mayolo.

\section{Fase de implementación de la metodología aula invertida}

En el año 2020, la asignatura de métodos numéricos se presentó utilizando un modelo de aula invertida, donde las clases presenciales fueron remplazadas por videos en YouTube, más una clase síncrona de tutoría a través de la plataforma Microsoft Teams. Se esperaba que los estudiantes vieran los videos antes de las clases cada semana. Las tutorías se utilizaron para interactuar con los estudiantes para despejar dudas, resolver interrogantes, realizar trabajos colaborativos y la resolución de problemas.

Las etapas realizadas en esta fase fueron: (1) al comienzo del semestre, se presentó a los estudiantes el paradigma del aula invertida y el temario del curso, asimismo se motivó al estudiante a ingresar a la plataforma donde se encontraba el material disponible de cada unidad; (2) Durante la metodología de trabajo, 
a los estudiantes se les encargó ver y analizar los videos correspondientes a la siguiente clase, antes de la misma, de manera que los estudiantes llegaran a la siguiente clase con los contenidos ya repasados. Con este estudio previo, se da inicio la clase síncrona indicando a los estudiantes que realicen la construcción del conocimiento a través de la formalización de los aprendizajes previamente revisados fuera del aula. En este mismo contexto, se procedió a aclarar las dudas y responder las preguntas que surgían de la revisión previa de los videos. Durante el cierre de la clase se indicó los videos que debían revisar para la clase siguiente. En el caso que los estudiantes no asistieran a clases presenciales, ellos desarrollaban las actividades desde su casa o lugar de trabajo para luego cargarlas al sistema en línea Moodle para la revisión y retroalimentación del profesor para estos estudiantes; (3) Se realizó una encuesta en línea al final, donde los 32 estudiantes respondieron preguntas respecto a la experiencia de la aplicación de la metodología de Aula invertida a través de la escala Likert, donde debían responder en una escala de cinco puntos: Muy en desacuerdo; en desacuerdo; neutral; De acuerdo y Totalmente de acuerdo.

\section{RESULTADOS Y DISCUSIÓN}

Hacia una medición del modelo de aula invertida sobre el rendimiento y la percepción, este trabajo se realizó en un curso de Métodos Numéricos, un total de 32 estudiantes participaron en el estudio.

\section{Análisis y evaluación del desempeño}

Para medir el rendimiento de los estudiantes en los tres años, usando rúbricas se evaluaron (en una escala de 0 a 20) tres actividades: las prácticas calificadas, investigación formativa y resolución de problemas a través de un video. Para el curso 2018, la tasa de aprobación fue del 81,58 \%, para el curso 2019 fue del 92,11 \% y para el curso 2020 fue del 94,29\%, lo que muestra la tendencia al aumento de los años anteriores, como se muestra en la tabla 1 (en los años 2018 y 2019 la metodología de enseñanza usada fue la metodología tradicional donde el profesor es quien enseña y dirige la clase, mientras los estudiantes son principalmente receptores). A partir de estos resultados, la tasa de aprobación del modelo de aula invertida superó la tasa de aprobación de años anteriores. Los resultados de la evaluación para el curso desarrollado con los mismos tópicos pero con diferente metodología de enseñanza-aprendizaje tal y como lo refleja la tabla 1. El promedio de calificaciones fue de 10 para 2018, 11 para 2019 y 14 para el año 2020. Como se observa en la tabla 1; hay diferencias considerables tanto en la tasa de reprobación la que disminuye entre los años como en el promedio de notas el que aumenta.

Tabla 1. Comparación de los resultados

\begin{tabular}{rccc}
\hline Criterio & Año 2018 & Año 2019 & Año 2020 \\
\hline N $^{\circ}$ Estudiantes & 38 & 35 & 35 \\
Promedio notas & 10 & 11 & 14 \\
Porcentaje de & 81,58 & 92,11 & 94,29 \\
$\begin{array}{r}\text { aprobación } \\
\text { Porcentaje de } \\
\text { desaprobados }\end{array}$ & 18 & 8 & 6 \\
\hline
\end{tabular}


Como se puede evidenciar en la tabla 1, los estudiantes alcanzan un promedio de calificaciones de 10 en año 2018 y 11 en el año 2019; mientras que en el año 2020 suben su rendimiento a 14 con lo cual se constata que la utilización del aula invertida es un método innovador que ayuda a mejorar el rendimiento académico. Este resultado está en concordancia con los encontrados por Vinay (2020). Los resultados demostraron que el modelo de aula invertida dio mejores resultados que las clases de años anteriores sin aplicar el modelo de aula invertida.

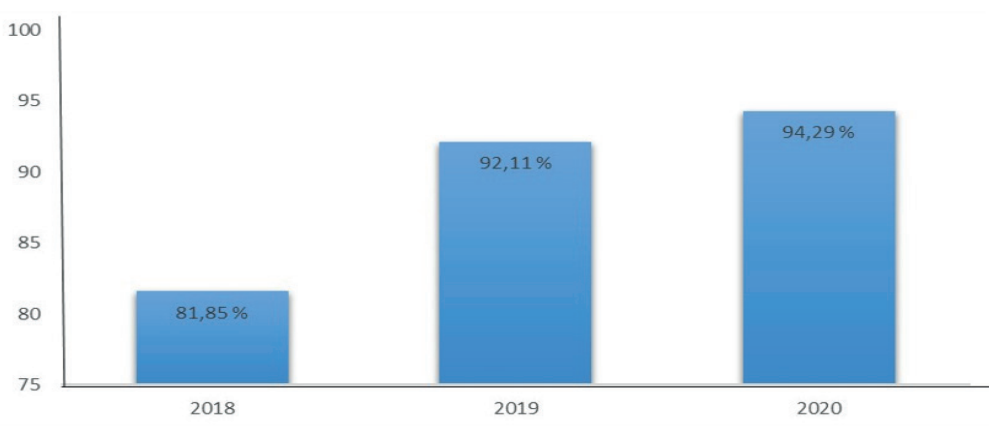

Figura 2. Tasa de aprobación asignatura de métodos numéricos entre el 2018 y el 2020

\section{Análisis de percepción y evolución}

Con respecto a la satisfacción de los estudiantes, la tabla 2 resume la información obtenida.

Los resultados que recogen las opiniones de los estudiantes mostraron que existía una percepción general positiva sobre el modelo de aula invertida empleado (ver figura 2). Casi el $56,25 \%$ de los estudiantes consideró que ver lecciones en video antes de asistir a clase era muy significativo para lograr los objetivos de aprendizaje. Dado que los materiales de las lecciones en video se diseñaron correctamente, bien estructurados y claramente definidos, la mayoría de los estudiantes estuvieron de acuerdo o muy de acuerdo con la declaración. El 56,25 \% de los estudiantes estuvo totalmente de acuerdo y el 37,50 $\%$ de ellos estuvo de acuerdo con la declaración. Las lecciones en video fueron muy instructivas y útiles para lograr los objetivos de aprendizaje.

Tabla 2. Resumen de satisfacción de estudiantes sobre la experiencia de metodología de aula invertida.

\begin{tabular}{|c|c|c|c|c|c|}
\hline Items & 1 & 2 & 3 & 4 & 5 \\
\hline $\begin{array}{l}\text { 1. La metodología de flipped classroom } \\
\text { ayuda a una mejor compresión de los } \\
\text { conceptos del curso }\end{array}$ & 3,13 & 3,13 & 12,50 & 78,13 & 3,13 \\
\hline $\begin{array}{l}\text { 2. La metodología de flipped classroom } \\
\text { es mejor que la clase tradicional }\end{array}$ & 0 & 12,5 & 28,13 & 50 & 9,38 \\
\hline $\begin{array}{l}\text { 3. La metodología de flipped classroom } \\
\text { motiva más para estudiar }\end{array}$ & 3,13 & 6,25 & 28,13 & 56,25 & 6,25 \\
\hline $\begin{array}{l}\text { 4. Con La metodología de flipped } \\
\text { classroom se ha mejorado el } \\
\text { aprendizaje }\end{array}$ & 3,13 & 3,13 & 28,13 & 56,25 & 9,38 \\
\hline $\begin{array}{l}\text { 5. Prefieres el método tradicional que } \\
\text { la metodología de flipped classroom }\end{array}$ & 6,25 & 50 & 28,13 & 15,63 & 0 \\
\hline $\begin{array}{l}\text { 6. Las clases grabadas fueron útiles } \\
\text { para mi aprendizaje. }\end{array}$ & 3,13 & 3,13 & 0 & 37,50 & 56,25 \\
\hline $\begin{array}{l}\text { 7. La mezcla de la clase síncrona y } \\
\text { asíncrona fue efectivo para el } \\
\text { aprendizaje de la asignatura. }\end{array}$ & 3,13 & 3,13 & 15,63 & 65,63 & 12,50 \\
\hline
\end{tabular}

Nota. $1=$ Totalmente en desacuerdo con el ítem; $2=$ En desacuerdo con el ítem; $3=$ Indiferente con el ítem, $4=$ De acuerdo con el ítem y $5=$ Totalmente de acuerdo con el ítem. 
De los resultados de la tabla 2 se desprende que la tasa de aprobación de la asignatura según diferentes periodos en los que se dictó la asignatura, en la cual se verifica un aumento en el porcentaje desde semestre año 2018 a 2019 en un 10,53 \%, y luego en el semestre 2020 un aumento de 2,18\% con respecto a al año 2019. Además, según la tabla 2, consideramos que la metodología de aula invertida ha sido bien recibida por los alumnos $(56,25 \%)$ y que la consideran más motivadora que la metodología tradicional. Este resultado está en consonancia con los encontrados previamente por Perdomo (2016).

De acuerdo con el modelo flipped classroom, previo a la clase, los estudiantes visualizan videos para apropiarse del contenido y aplicarlo en el aula. Al respecto, se puede observar una percepción positiva $(37,50 \%$ de acuerdo y 56,25 \% totalmente de acuerdo) por parte del estudiantado, ya que pueden detener el video, adelantarlo y atrasarlo cuantas veces lo deseen, permitiendo con ello una mejor comprensión del contenido y un ajuste de la metodología a los distintos ritmos y estilo de aprendizaje de los estudiantes, lo cual según Bergmann y Sams (2012) se logra dado que el flipped classroom promueve una enseñanza más personalizada y se atienden las necesidades particulares de los estudiantes en clase (Tourón y Santiago, 2015).

Cuando se interroga al alumnado acerca de qué metodología prefieren en clase, la mayoría se manifiesta a favor de las clases invertidas frente a las clases tradicionales (Chin, 2014; Galway, Corbett, Takaro, Tairyan, y Frank, 2014). Esto concuerda con los resultados donde el $50 \%$ de los estudiantes estuvieron en desacuerdo a la afirmación "Prefieres el método tradicional de cla- ses que con la metodología aula invertida".

Asimismo, los resultados de esta investigación coinciden con Fulton (2012) al indicar que los estudiantes deben hacer la preparación requerida mucho antes de llegar al aula, de manera similar con Sever (2014) al afirmar que, en el aula invertida, los estudiantes deben tener conocimientos previos antes de la hora de clase para aumentar el éxito. Por lo tanto, el hecho de que los estudiantes estén listos para hacer avances significa que tienen una alta preparación para el aula invertida. Esto confirma que de los estudiantes participantes $56,25 \%$ estuvieron de acuerdo con que la metodología de flipped classroom motiva más para estudiar, por lo que es posible decir que su preparación para el aula invertida es positiva.

En general, los estudiantes se sintieron más seguros de su capacidad para aprender matemáticas de forma independiente, mostraron una mejor retención de los materiales a lo largo del tiempo y disfrutaron de la experiencia invertida, con el sistema de evaluación, y la posibilidad de ser entes activos en su aprendizaje. A pesar de que el tiempo y las habilidades digitales resultaron ser los mayores retos para cumplir con los objetivos planteados, las experiencias didácticas vividas y compartidas por los participantes sirvieron de herramientas para su vinculación y entusiasmo en un entorno de aprendizaje más auténtico. El aula invertida ofrece oportunidades para estimular e involucrar a los alumnos en su proceso de enseñanza y aprendizaje. Entre estas se destacan las siguientes: indagar sobre los contenidos a desarrollar en clase, utilizar herramientas tecnológicas para maximizar el tiempo, interactuar y ser más efectivos, tener un rol activo en el aprendizaje al participar con ímpetu en las actividades 
programadas dentro y fuera del aula, desarrollar las destrezas y habilidades que le permiten ser aprendices más motivados y autónomos tal como lo indica Galway et. al., (2014).

\section{CONCLUSIONES}

La aplicación del modelo de aula invertida fue realizada con videos tutoriales, para que estos estudiantes pudieran revisar en el momento que ello desee y lograr aprendizajes significativos, desarrollando así un aprendizaje autónomo y regulado, los cuales tuvieron un impacto positivo en su retención, aprobación y promedio de notas. Los estudiantes a través de una encuesta, catalogaron positivamente su experiencia sobre un aula invertida, ya que lograron estudiar a su propio ritmo, repitiendo la cla- se si fuera necesario y teniendo acceso a la misma desde cualquier lugar, lo que aumentó su motivación y satisfacción con la asignatura de Métodos Numéricos, favoreciendo el aprendizaje activo y significativo.

La experiencia llevada a cabo ha sido exitosa en cuanto a grado de satisfacción de los alumnos y, por lo tanto, podemos recomendar generalizar su implementación en otros cursos y temas de matemática. Además, la metodología de clase invertida es una alternativa para el desarrollo de competencias en estudiantes en estos tiempos de crisis sanitaria en que vivimos, y representa una alternativa adecuada para el desarrollo de competencias y aprendizajes esenciales en la asignatura de Métodos Numéricos.

\section{REFERENCIAS BIBLIOGRÁFICAS}

Arráez Vera, G., Lorenzo Lledó, A., Gómez Puerta, M., y Lorenzo Lledó, G. (2018). La clase invertida en la educación superior: percepciones del alumnado. Revista INFAD de Psicología. International Journal of Developmental and Educational Psychology, 2(1), 155-162. doi:https://doi.org/10.17060/ijodaep.2018.n1.v2.1197

Bergmann, J., y Sams, A. (2014). Flipped learning: Gateway to student engagement. International Society for Technology in Education. https://dspacecdc-test.inlibro.net/xmlui/handle/11515/34587

Bergmann, J., y Sams, A. (2012). Flip your classroom: Reach every student in every class every day. International Society for Technology in Education. http://www.daneshnamehicsa. ir/userfiles/files/1/17-\%20Flip\%20Your\%20Classroom_\%20Reach\%20Every\%20Student $\% 20$ in $\% 20$ Every\%20Class\%20Every\%20Day\%20(ASCD).pdf

Bolat, Y. (2016). The flipped classes and education information network (EIN) Ters yüz edilmiş sınıflar ve eğitim bilişim ağı (EBA). Journal of Human Sciences, 13(2), 3373-3388. https://www.j-humansciences.com/ojs/index.php/IJHS/article/view/3952

Bullock, S. M. (2013). Using Digital Technologies to Support Self-Directed Learning for Preservice Teacher Education. https://doi.org/10.1080/09585176.2012.744695 
Chin, C. A. (2014). Evaluation of a flipped classroom implementation of Data Communications Course: Challenges, insights and suggestions. In SOTL 2014 Proceedings. http://www.spsu.edu/cte/publications/publications2014/sotl_2014_chin.pdf.

DeSantis, J. V. (2015). Do Students Learn More from a Flip? An Exploration of the Efficacy of Flipped and Traditional Lessons. Journal of Interactive Learning Research, 26(1), 39-63. https://www.learntechlib.org/primary/p/130133/.

DeLozier, S. J., y Rhodes, M. G. (2016). Flipped Classrooms: a Review of Key Ideas and Recommendations for Practice. Educational Psychology Review, 29, 1-11. https://doi.org/10.1007//10648-015-9356-9.

Carpenter, R. G., Sweet, C., Blythe, H., Winter, R., y Bunnell, A. (2015). A Challenge for the Flipped Classroom: Addressing Spatial Divides. In A. Scheg (Ed.), Implementation and Critical Assessment of the Flipped Classroom Experience (pp. 139-156). IGI Global. http://doi:10.4018/978-1-4666-7464-6.ch008

Dodds, M. (2015). Evidence for the Flipped Classroom in STEM. York University PGCHE Resources. http://citeseerx.ist.psu.edu/viewdoc/download?doi=10.1.1.696.1252\&rep=rep1\&type $=$ pdf $[12-06-2020]$

Filiz, O., y Kurt, A. A. (2015). Flipped learning: Misunderstandings and the truth. Journal of Educational Sciences Research, 5(1), 215-229. https://www.academia.edu/11970218/Flipped_Learning_Misunderstandings_and_the_Truth

Freeman, S., Eddy, S. L., McDonough, M., Smith, M. K., Okoroafor, N., Jordt, H., y Wenderoth, M. P. (2014). Active learning increases student performance in science, engineering, and mathematics. Proceedings of the National Academy of Sciences, 111(23), 8410-8415. https://doi.org/10.1073/pnas.1319030111

Fox-Turnbull, W. H., Docherty, P., y Zaka, P. (2018). Learning engineering through the Flipped Classroom Approach- students' perspectives. Design and Technology Education: An International Journal, 23(3), 27-45. https://ojs.lboro.ac.uk/DATE/article/view/2470/2730

Fulton, K. (2012). Inside the flipped classroom. The Journal, 4(11). https://thejournal.com/articles/2012/04/11/the-flipped-classroom.aspx

Galway, L. P., Corbett, K. K., Takaro, T. K., Tairyan, K., y Frank, E. (2014). A novel integration of online and flipped classroom instructional models in public health higher education. BMC medical education, 14(1), 181. https://bmcmededuc.biomedcentral.com/articles/10.1186/1472-6920-14-18 
García, J. (2020). Tecnología como herramienta. Con-Ciencia Boletín Científico De La Escuela Preparatoria No. 3, 7(13), 40-41. https://repository.uaeh.edu.mx/revistas/index.php/prepa3/article/view/5201

García, M., Porto, M., y Hernández, F. (2019). El aula invertida con alumnos de primero de magisterio: fortalezas y debilidades. REDU. Revista de Docencia Universitaria, 17(2), 89-106.

https://polipapers.upv.es/index.php/REDU/article/view/11076/12228.

Gilboy, M.B., Heinerichs, S. y Pazzaglia, G. (2015). Enhancing Student Engagement Using the Flipped Classroom. Journal of Nutrition Education and Behavior. (47) 1. 109-114 https://doi.org/10.1016/j.jneb.2014.08.008.

Jungić, V., Kaur, H., Mulholland, J. y Xin, C. (2015). On flipping the classroom in large first year calculus courses. International Journal of Mathematical Education in Science and Technology, 46(4), 508-520, doi: 10.1080/0020739X.2014.990529

Little, C. (2015). The Flipped Classroom in Further Education: Literature Review and Case Study. Research in Post-Compulsory Education. 20(3), 265-279, doi: $10.1080 / 13596748.2015 .1063260$

Maciejewski, W. (2015) Flipping the calculus classroom: an evaluative study. Teach. Math. Appl., 1-15. doi: 10.1093/teamat/hrv019

Mastmeyer, A. (2020). Quantitative and Qualitative Evaluation of Transforming to Flipped-Classroom from Instruction Teaching Using Micro Feedback Loops. Online Submission. https://files.eric.ed.gov/fulltext/ED607324.pdf

Novak, J., Kensington-Miller, B., y Evans, T. (2017). Flip or flop? Students' perspectives of a flipped lecture in mathematics. International Journal of Mathematical Education in Science and Technology, 48(5), 647-658. doi: 10.1080/0020739X.2016.1267810

Petrillo, J. (2016). On flipping first-semester calculus: a case study. International Journal of Mathematical Education in Science and Technology, 47(4), 573-582, doi: 10.1080/0020739X.2015.1106014

Rivera, F. M., y García, A. (2018). Aula invertida con tecnologías emergentes en ambientes virtuales en la Universidad Politécnica Salesiana del Ecuador. Revista Cubana de Educación Superior, 37(1), 108-123. Recuperado de http://scielo.sld.cu/scielo.php?script=sci_arttext\&pid=S0257-43142018000100008. 
Rotellar, C., y Cain, J. (2016). Research, Perspectives, and Recommendations on Implementing the Flipped Classroom. American Journal of Pharmaceutical Education, 80(2), 34. https://doi.org/10.5688/ajpe80234.

Tourón, J., y Santiago, R. (2015). El modelo Flipped Learning y el desarrollo del talento en la escuela. Revista de Educacion, (368), 33-65. doi:10.4438/1988-592X-RE-2015-368-288

Torun, F., y Dargut, T. (2015). A proposal for the applicability of flipped classroom model in mobile learning environments. Journal of Educational Sciences, 6(2), 20-29. https://core.ac.uk/download/43802180.pdf

Perdomo, W. (2016). Estudio de evidencias de aprendizaje significativo en un aula bajo el modelo Flipped Classroom. Edutec. Revista Electrónica De Tecnología Educativa, (55), 325. https://doi.org/10.21556/edutec.2016.55.618

Vinay, A. (2020). Action Research in Education: Effectiveness of Flipped Classroom on Academic Performance of Students. Online Submission. https://files.eric.ed.gov/fulltext/ED607521.pdf 